\title{
Integration of Single Oriented Oxide Superlattices on Silicon Using Various Template Techniques
}

\author{
Binbin Chen, Zoran Jovanovic, Stefan Abel, Phu Tran Phong Le, Ufuk Halisdemir, Mark Smithers, \\ Daniel Diaz-Fernandez, Matjaž Spreitzer, Jean Fompeyrine, Guus Rijnders, and Gertjan Koster*
}

Cite This: ACS Appl. Mater. Interfaces 2020, 12, 42925-42932

Read Online

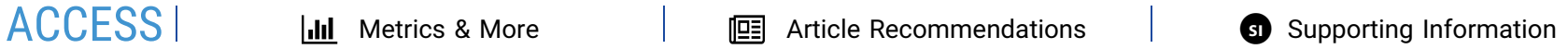

ABSTRACT: To benefit from the diverse functionalities of perovskite oxides in silicon-based complementary metal oxide semiconductor (CMOS) technology, integrating oxides into a silicon platform has become one of the major tasks for oxide research. Using the deposition of $\mathrm{LaMnO}_{3} / \mathrm{SrTiO}_{3}$ (STO) superlattices (SLs) as a case study, we demonstrate that (001) single oriented oxide SLs can be integrated on Si using various template techniques, including a single-layer buffer of STO prepared by molecular beam epitaxy (MBE) and pulsed laser deposition, a multilayer buffer of Y-stabilized zirconia/ $\mathrm{CeO}_{2} /$ $\mathrm{LaNiO}_{3} / \mathrm{STO}$, and STO-coated two-dimensional nanosheets of $\mathrm{Ca}_{2} \mathrm{Nb}_{3} \mathrm{O}_{10}$ (CNO) and reduced graphene oxide. The textured SL grown on STO-coated $\mathrm{CNO}$ nanosheets shows the highest

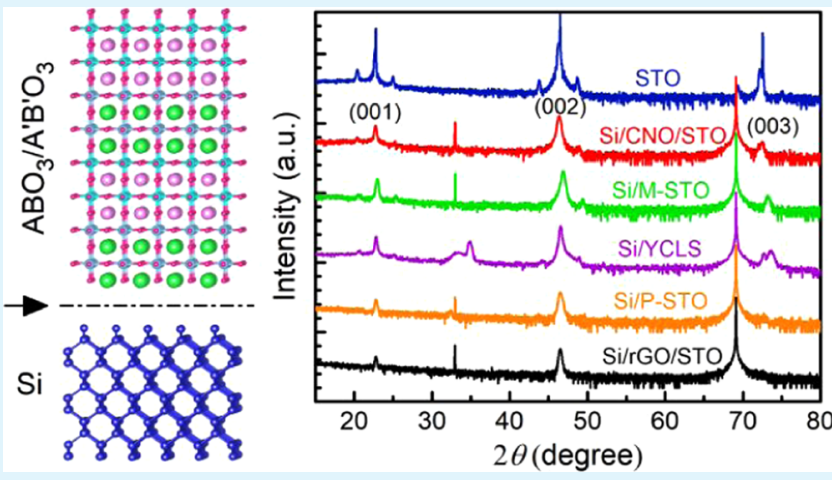
crystallinity, owing to the small lattice mismatch between CNO and STO as well as less clamping from a Si substrate. The epitaxial SL grown on STO buffer prepared by MBE suffers the largest thermal strain, giving rise to a strongly suppressed saturation magnetization but an enhanced coercive field, as compared to the reference SL grown on an STO single crystal. These optional template techniques used for integrating oxides on $\mathrm{Si}$ are of significance to fulfill practical applications of oxide films in different fields.

KEYWORDS: oxide superlattice, silicon, template, epitaxy, magnetism

\section{INTRODUCTION}

Transition metal oxides adopting a perovskite structure hold a wide spectrum of properties governed by the ubiquitous electron-electron and electron-lattice interactions. ${ }^{1}$ Typical examples include ferromagnetism, ferro/piezoelectricity, multiferroicity, colossal magnetoresistance, superconductivity, etc. ${ }^{2}$ Their similar crystal structure enables the assembly of heterostructures and superlattices with coherent lattices and chemically abrupt interfaces. In such architectures, oxide properties can be further tuned by the interface effects, such as epitaxial strain, charge transfer, electron confinement, oxygen octahedral coupling, and orbital hybridization. ${ }^{3-6}$ Extensive research has been undertaken to integrate functional oxides into a silicon platform aiming at enhancing the performance of next-generation nanoelectronic devices. ${ }^{7,8}$ For instance, ferroelectric oxides are explored as the gating layer in metal-oxide-semiconductor (MOS) field-effect transistors for nonvolatile memories. ${ }^{8}$ However, the epitaxy of oxides on silicon remains a great challenge given the structural difference, large thermal mismatch, and propensity to form amorphous $\mathrm{SiO}_{2}$ at the interface. ${ }^{7,8}$

The integration of oxides on silicon was initially motivated by the searching of high- $k$ oxides to replace $\mathrm{SiO}_{2}$ in $\mathrm{MOS}$ transistors for a continuous device downscaling. ${ }^{9}$ Later on, some of these oxides, such as $\mathrm{Y}_{2} \mathrm{O}_{3},{ }^{10} \mathrm{Al}_{2} \mathrm{O}_{3},{ }^{11} \mathrm{MgO},{ }^{12}$ and yttria-stabilized zirconia (YSZ), ${ }^{13}$ were also explored as buffer layers for the overgrowth of other functional oxides on $\mathrm{Si}$. Nevertheless, the epitaxy of perovskite oxides on such buffer layers is still not straightforward due to their large structural differences. A breakthrough was made by McKee and coworkers in the 90s, who first demonstrated that $\mathrm{SrTiO}_{3}$ (STO) can be grown directly on silicon with an atomically sharp interface using molecular beam epitaxy $(\mathrm{MBE}){ }^{14}$ Thereafter, significant progress has been made based on an STO template, thanks to its closely matched lattice constants with most perovskite oxides. For instance, piezoelectric/flexoelectric microelectromechanical systems, ${ }^{15,16}$ electrically switchable two-dimensional electron gas, ${ }^{17}$ and $\mathrm{BaTiO}_{3}$-based electricaloptical devices $^{18,19}$ have been demonstrated on the Si/STO

Received: June 15, 2020

Accepted: August 26, 2020

Published: August 26, 2020 

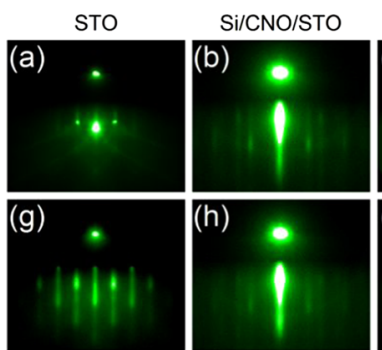
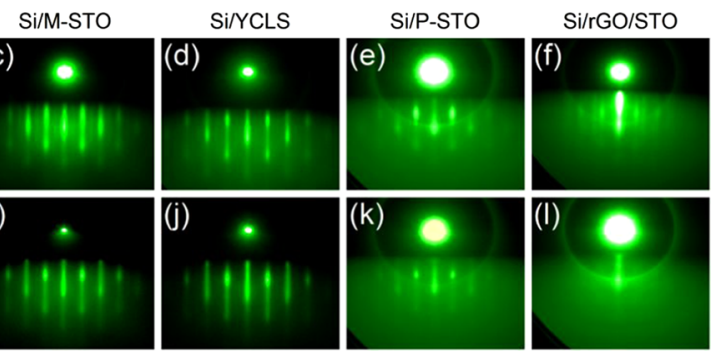

Figure 1. RHEED patterns acquired prior to $(a-f)$ and after $(g-l)$ the growth of LMO/STO SLs on the STO single crystal (a, g), Si/CNO/STO (b, h), Si/M-STO (c, i), Si/YCLS (d, j), Si/P-STO (e, k), and Si/rGO/STO (f, l). The patterns were taken along STO [100] and Si [110] azimuths, respectively.

pseudosubstrate during the past decade. Given the fact that MBE requires ultrahigh vacuum and gives a very slow growth rate, people are also seeking for more cost-effective techniques to make the STO template on silicon, such as magnetron sputtering and pulsed laser deposition (PLD). ${ }^{20-23}$ Moreover, two-dimensional templates like oxide nanosheets and graphene have also been applied to grow highly oriented oxide films on silicon. $^{24-26}$

A heterointerface between STO and $\mathrm{LaMnO}_{3}$ (LMO) has gained considerable interest for the thickness-dependent electronic reconstructions as well as the ferromagnetic insulating character. ${ }^{27-29}$ In this paper we show that (001) single oriented LMO/STO superlattices (SLs) can be integrated on a $\mathrm{Si}$ substrate using various template techniques, including a single-layer buffer of STO fabricated by $\mathrm{MBE}$ and PLD (hereafter, shorted as M-STO and P-STO), YSZ/ $\mathrm{CeO}_{2} /$ $\mathrm{LaNiO}_{3} / \mathrm{STO}$ (YCLS) multilayer buffer, STO-coated twodimensional nanosheets of $\mathrm{Ca}_{2} \mathrm{Nb}_{3} \mathrm{O}_{10}(\mathrm{CNO} / \mathrm{STO})$, and reduced graphene oxide (rGO/STO). The SLs on M-STO, PSTO, and YCLS show an epitaxial relationship with the $\mathrm{Si}$ substrate, while in-plane textured films are obtained on $\mathrm{CNO} /$ STO and rGO/STO. The SL on CNO/STO exhibits the highest crystallinity with a rocking curve full-width at halfmaximum (FWHM) of $0.18^{\circ}$, significantly smaller than the ones on M-STO $\left(\sim 0.88^{\circ}\right)$, YCLS $\left(\sim 1.14^{\circ}\right)$, P-STO $\left(2.66^{\circ}\right)$, and $\mathrm{rGO} / \mathrm{STO}\left(2.36^{\circ}\right)$. As compared to the reference SL grown on an STO single crystal, the ferromagnetic properties are modified by defects and strain for the SLs grown on silicon.

\section{EXPERIMENTAL SECTION}

Preparation of Various Templates on Silicon. M-STO. MBE deposition was carried out in a chamber with a base pressure below 3 $\times 10^{-10}$ mbar. After removing the native $\mathrm{SiO}_{2}$ by HF-etching, the $\mathrm{Si}$ substrate was heated to $600-650{ }^{\circ} \mathrm{C}$ and a half monolayer of Sr was deposited to passivate the active Si surface. After cooling down to 50 ${ }^{\circ} \mathrm{C}$, the sample was exposed to molecular oxygen to oxidize the $\mathrm{Sr}$ layer. This was followed by the deposition of 10 unit cells (uc) STO in $5 \times 10^{-7} \mathrm{mbar}_{2}$. Afterward, the amorphous STO layer was annealed at $400-500^{\circ} \mathrm{C}$ in ultrahigh vacuum to obtain crystalline and epitaxial STO on silicon. More details can be found in previous papers. $^{19,30}$

P-STO. STO was grown in a PLD chamber with a base pressure around $8 \times 10^{-9} \mathrm{mbar}$. The silicon substrate was first baked at $630{ }^{\circ} \mathrm{C}$ for $12 \mathrm{~h}$ for a thorough degassing, followed by a flash annealing at $1200{ }^{\circ} \mathrm{C}$ for $1.5 \mathrm{~min}$ to remove the native oxide. Half monolayer of $\mathrm{Sr}$ was then deposited at $700{ }^{\circ} \mathrm{C}$ using a laser fluence of $1 \mathrm{~J} / \mathrm{cm}^{2}$ and a frequency of $0.25 \mathrm{~Hz}$. After cooling down to room temperature, $2 \mathrm{uc}$ STO was deposited in $5 \times 10^{-2} \mathrm{mbar}$ Ar using a laser fluence of $1.5 \mathrm{~J} /$ $\mathrm{cm}^{2}$ and a frequency of $1 \mathrm{~Hz}$, followed by an exposure to oxygen to improve the stoichiometry. The deposition procedure of STO was repeated four more times to reach a layer thickness of $10 \mathrm{uc}$. The amorphous STO was then annealed in ultrahigh vacuum at $515^{\circ} \mathrm{C}$ for 15 min to get crystallized. Another 90 uc STO was deposited on $\mathrm{Si} /$ STO $(10 \mathrm{uc})$ at $515{ }^{\circ} \mathrm{C}$ in $1.3 \times 10^{-6} \mathrm{mbar}_{2}$. More details can be found in previous papers. ${ }^{21-23}$

YCLS. The YCLS multilayer was prepared by PLD in situ monitored by reflection high-energy electron diffraction (RHEED). Polycrystalline YSZ $\left(\mathrm{ZrO}_{2}-8 \mathrm{~mol} \% \mathrm{Y}_{2} \mathrm{O}_{3}\right), \mathrm{CeO}_{2}, \mathrm{LaNiO}_{3}$, and single-crystalline STO targets were ablated using a laser fluence of $2 \mathrm{~J} / \mathrm{cm}^{2}$ and a frequency of $2 \mathrm{~Hz}$. The YSZ layer was grown directly on $\mathrm{Si}$ at $800{ }^{\circ} \mathrm{C}$ without removing native oxides. The first $\sim 10 \mathrm{~nm}$ YSZ was deposited in 0.02 mbar Ar to facilitate the scavenge reaction, ${ }^{31}$ then another $\sim 10 \mathrm{~nm} \mathrm{YSZ}$ and $\sim 8 \mathrm{~nm} \mathrm{CeO}_{2}$ were successively deposited in $4 \times$ $10^{-4} \mathrm{mbar}_{2}$. After cooling down to $700{ }^{\circ} \mathrm{C}$, perovskite $\mathrm{LaNiO}_{3}(\sim 8$ $\mathrm{nm})$ and STO $(\sim 40 \mathrm{~nm})$ layers were deposited in $0.15 \mathrm{mbar}_{2}$. RHEED patterns recorded after the growth of each layer are shown in Figure S1 of the Supporting Information.

CNO/STO. We deposited CNO nanosheets on Si using LangmuirBlodgett (LB) method. The CNO nanosheets were obtained through exfoliation from protonated calcium niobite, $\mathrm{HCa}_{2} \mathrm{Nb}_{3} \mathrm{O}_{10} \cdot 1.5 \mathrm{H}_{2} \mathrm{O}$. The LB deposition was conducted in a double-barrier Langmuir trough under a constant rate of compression $(3.0 \mathrm{~mm} / \mathrm{min})$. After immersing the $\mathrm{Si}$ substrate into the dispersion of $\mathrm{CNO}$ nanosheets, the barriers started to move until a required surface pressure $(17 \mathrm{mN} /$ m) for a high coverage was achieved. Then, the Si substrate was lifted out of the dispersion at a rate of $1 \mathrm{~mm} / \mathrm{min}$, during which $\mathrm{CNO}$ nanosheets were transferred to the Si surface. More details can be found in our previous papers. ${ }^{25,32}$ The STO layer of $40 \mathrm{~nm}$ thickness was deposited by PLD at $700{ }^{\circ} \mathrm{C}$ in $0.2 \mathrm{mbar}_{2}$. The laser fluence and frequency were set to $2 \mathrm{~J} / \mathrm{cm}^{2}$ and $2 \mathrm{~Hz}$, respectively. Layer-bylayer growth of STO on CNO nanosheets was observed from RHEED (Figure S2 in the Supporting Information).

rGO/STO. Graphene oxide nanosheets were first obtained from the chemical exfoliation of graphite. The nanosheets dispersed in water were then deposited on the Si substrate via a spin coating method. The $\mathrm{Si} / \mathrm{rGO}$ substrate was heated to $650{ }^{\circ} \mathrm{C}$ in high vacuum and annealed for $2 \mathrm{~h}$ in a PLD chamber. To deoxidize the Si surface uncovered by rGO and achieve a (001) single oriented growth of STO, 15 pulses of SrO were first deposited using a laser fluence of 2 $\mathrm{J} / \mathrm{cm}^{2}$ and a frequency of $0.1 \mathrm{~Hz}^{33}$ After flash annealing at $840{ }^{\circ} \mathrm{C}$ for $2 \mathrm{~min}$, the sample was cooled down to $760^{\circ} \mathrm{C}$ and $40 \mathrm{~nm} \mathrm{STO}$ was deposited in high vacuum with a laser fluence of $2 \mathrm{~J} / \mathrm{cm}^{2}$ and a frequency of $3 \mathrm{~Hz}$. RHEED patterns recorded during the growth of $\mathrm{SrO}$ and STO can be found in Figure S3 of the Supporting Information.

Growth of LMO/STO SLs. LMO/STO SLs were synthesized using PLD in situ monitored by RHEED. The layer thicknesses of LMO and STO were set to 6 and 4 uc, respectively. The bilayer was repeated 10 times to form the SL. Polycrystalline LMO and singlecrystalline STO targets were ablated using a laser fluence of $2 \mathrm{~J} / \mathrm{cm}^{2}$ with a spot size of $2.2 \mathrm{~mm}^{2}$. The frequency was set to $2 \mathrm{~Hz}$. During the deposition, the substrate temperature and oxygen pressure were set at $700{ }^{\circ} \mathrm{C}$ and $0.01 \mathrm{mbar}$, respectively. The SLs were in situ annealed for $10 \mathrm{~min}$ before cooling down to room temperature in the deposition pressure at a ramping rate of $10{ }^{\circ} \mathrm{C} / \mathrm{min}$. 


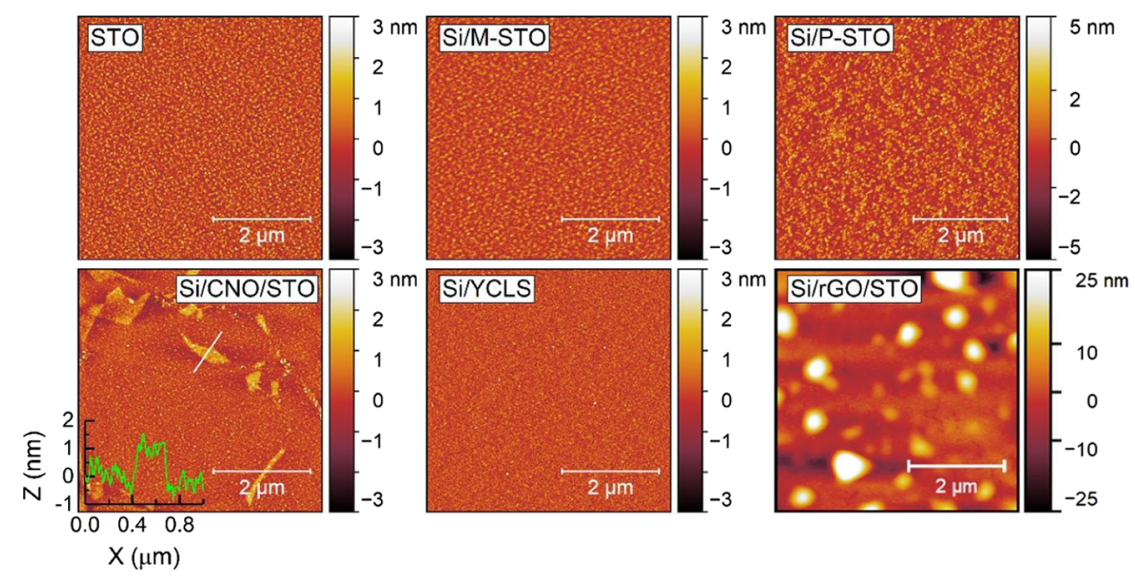

Figure 2. AFM images of LMO/STO SLs grown on various substrates. The height profile along the white line is shown in the inset for the SL on $\mathrm{Si} / \mathrm{CNO} / \mathrm{STO}$.
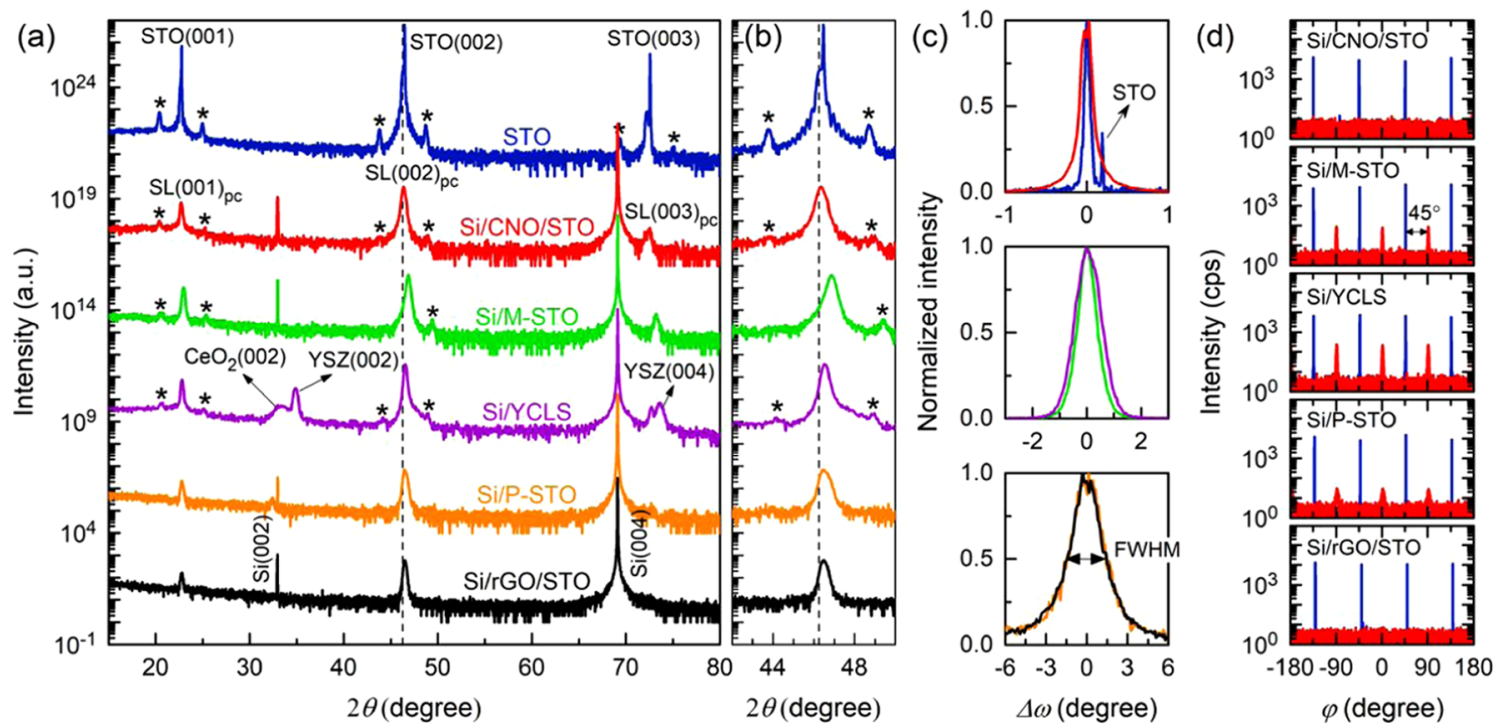

Figure 3. XRD $\theta-2 \theta$ scans (a), zoom of the $(002)_{\mathrm{pc}}$ peak (b), rocking curves around the (002) $)_{\mathrm{pc}}$ peak (c), and $\varphi$ scans of $\mathrm{Si}(202)$ and $\mathrm{SL}(101)_{\mathrm{pc}}$ reflection (d) measured from LMO/STO SLs grown on various substrates. The satellite peaks are labeled with asterisks in $(\mathrm{a}, \mathrm{b})$. (d) $\varphi$ Scans of $\mathrm{Si}$ and SL are plotted in blue and red, respectively.

Sample Characterization. The surface morphology was characterized using Bruker atomic force microscopy (AFM). The crystal structure was studied by high-resolution X-ray diffraction (XRD) performed on a PANalytical-X'pert material research diffractometer, where the 4-bounce $\mathrm{Ge}(220)$ monochromator gives an angle divergence of $\sim 0.005^{\circ}$. The magnetic properties were measured using vibrating sample magnetometry (VSM) on a Quantum Design physical property measurement system (PPMS).

\section{RESULTS AND DISCUSSION}

Figure 1 shows RHEED patterns acquired prior to and after the growth of LMO/STO SLs on silicon with various buffer layers. The SL grown on an STO single-crystal substrate is included as a benchmark. Clear RHEED oscillations were observed within the first three SL periods for LMO/STO SLs grown on STO and Si/M-STO (Figure S4 of the Supporting Information), from which the growth rates of LMO and STO layers were calibrated. ${ }^{34}$ As can be seen from Figure 1a,g, the treated STO substrate with $\mathrm{TiO}_{2}$-single termination shows sharp spots with Kikuchi lines, while the spots turn into streaks with a modulated intensity after the growth of LMO/STO SL. Such intensity modulation is due to electron transmission through small grains on the surface, indicative of surface roughening. ${ }^{35}$ For the $\mathrm{CNO} / \mathrm{STO}$ template, STO layer grows in a layer-by-layer manner because of the small lattice mismatch between CNO $(a=3.86 \AA)$ and STO $(a=3.905$ $\AA$ ) (Figure S2 of the Supporting Information), resulting in a smooth surface exhibiting a sharp and streaky specular spot (Figure 1b). Besides, extra streaks can be discerned. They cannot be ascribed to the coherent diffraction from a single STO grain, which gives streaks with equal spacing. Instead, such streaks originate from the alignment of multiple STO grains with RHEED beam along different crystal axes, given the in-plane randomly oriented $\mathrm{CNO}$ nanosheets along with the relatively large RHEED beam size as compared to the typical dimensions of the nanosheets. ${ }^{25}$ The pattern remains essentially unchanged after the deposition of LMO/STO SL (Figure $1 \mathrm{~h}$ ), signifying a two-dimensional growth mode. The M-STO, YCLS, and P-STO templates show similar features with intensity-modulated streaks (Figure $1 \mathrm{c}-\mathrm{e}$ ). After the growth of SL, the patterns are slightly altered and reminiscent of the reference SL on the STO single crystal (Figure 1i-k). It is noteworthy that the intensity modulation along streaks is less 
evident for the SL grown on YCLS (Figure 1j), indicative of a smoother surface. Moreover, the patterns are much more diffuse for the SL on P-STO (Figure 1e,k) because of more crystalline disorders. ${ }^{36}$ The $\mathrm{rGO} / \mathrm{STO}$ template shows a sharp specular spot and additional streaks (Figure 1f), resembling CNO/STO. Such streaks are hardly visible after the deposition of LMO/STO SL and the pattern becomes more blurred (Figure 11), corresponding to the degradation of crystallinity. ${ }^{36}$

The above RHEED analyses are further supported by AFM characterizations, as shown in Figure 2. The SLs grown on an STO single crystal, $\mathrm{Si} / \mathrm{M}$-STO, and $\mathrm{Si} / \mathrm{P}-\mathrm{STO}$ show relatively smooth surfaces with root-mean-square ( $\mathrm{rms}$ ) roughness of $\sim 5.5, \sim 4.5$, and $\sim 9.5 \AA$, respectively. Their grainy features are in accordance with the modulated streaks observed from RHEED (Figure 1g,i,k). By contrast, the surface is less grainy for the SL grown on YCLS, and a smaller rms roughness of $\sim 3.9 \AA$ is achieved. Remarkably, the SL on Si/CNO/STO is so flat that the thickness variation $(\sim 1 \mathrm{~nm})$ across a single CNO nanosheet can be appreciated, as inferred from the line profile shown in the inset. A rms roughness of $\sim 5 \AA$ is determined for the scanning area. The $\mathrm{SL}$ on $\mathrm{Si} / \mathrm{rGO} / \mathrm{STO}$ is rough with large particles on the surface. Such particles are formed during the deposition of LMO/STO SL in an oxygen atmosphere (0.01 mbar) since they are not observed after the deposition of 40 $\mathrm{nm}$ STO on rGO nanosheets in high vacuum.

We focus on the crystal structures of LMO/STO SLs characterized using high-resolution XRD. As can be seen from XRD $\theta-2 \theta$ scans depicted in Figure 3a, all SLs show only (00l) peaks without secondary phases being detected, attesting to single oriented and phase pure samples. In particular, it has been demonstrated that STO grown on a graphene buffer layer on silicon has a dominant $(00 l)$ orientation, but coexisting with a small portion of (110)-, (111)-, and (211)-oriented grains. ${ }^{26}$ In this work, we employ $\mathrm{SrO}$ to deoxidize the uncovered $\mathrm{Si}$ surface before STO deposition, ${ }^{33}$ which plays a key role in inducing (001) single oriented growth of STO on rGO. The details will be presented in a separate publication. As marked by asterisks in Figure 3a,b, satellite peaks are observed for the SLs grown on STO, Si/CNO/STO, Si/M-STO, and Si/YCLS, but absent for the SLs on $\mathrm{Si} / \mathrm{P}-\mathrm{STO}$ and $\mathrm{Si} / \mathrm{rGO} / \mathrm{STO}$. The presence of satellite peaks indicates a well-defined composition modulation along the growth direction as well as sharp interfaces between the component layers. The SL period calculated from those satellite peaks is $3.8 \pm 0.1 \mathrm{~nm}$, in good agreement with the expected value of $\sim 3.9 \mathrm{~nm}$. Figure $3 \mathrm{c}$ shows the rocking curves around $(002)_{\mathrm{pc}}(\mathrm{pc}$, pseudocubic) peaks of LMO/STO SLs. The SL on $\mathrm{Si} / \mathrm{CNO} / \mathrm{STO}$ shows a FWHM of $\sim 0.18^{\circ}$, slightly larger than the reference SL on STO single crystal showing a FWHM of $\sim 0.08^{\circ}$. For comparison, the smallest FWHM reported for oxide films grown on nanosheets is $0.10^{\circ} .25$ The high crystallinity can be attributed to the small lattice mismatch between oxides and $\mathrm{CNO}$ as well as less clamping effects from the Si substrate, as we will discuss later. Combining with the rather smooth surface, $\mathrm{CNO} / \mathrm{STO}$ can be an ideal template to integrate highly oriented and crystalline oxide films on silicon, ${ }^{25,32}$ although they are in-plane textured, as we will show in the following. The SLs grown on Si/M-STO and Si/YCLS show FWHM of $\sim 0.88$ and $\sim 1.14^{\circ}$, nearly 10 times larger than the ones on STO and $\mathrm{Si} / \mathrm{CNO} / \mathrm{STO}$. We notice that the crystalline quality of our SLs is comparable to ferroelectric $\mathrm{BaTiO}_{3}$ films recently reported on $\mathrm{Si} / \mathrm{M}-\mathrm{STO}$ and $\mathrm{Si} /$ YCLS substrates showing typical FWHM of 0.84 and $1.10^{\circ} .^{37,38}$ It should be noted that the SL on YCLS buffer was fabricated by an entirely in situ process using PLD, providing an advantage over M-STO buffer. For instance, the surface adsorbates can be largely avoided when using YCLS templates for the PLD growth of oxide materials at a relatively low temperature. The SLs grown on $\mathrm{Si} / \mathrm{P}-\mathrm{STO}$ and $\mathrm{Si} / \mathrm{rGO} / \mathrm{STO}$ show broad rocking curves with FWHM of $\sim 2.66$ and $\sim 2.36^{\circ}$, respectively, indicating relatively poor crystallinity. The results are in line with the faint RHEED patterns, as shown in Figure 1k,l, as well as the cross-sectional scanning electron microscopy characterizations, as shown in Figure S5 of the Supporting Information. To confirm the in-plane epitaxial relationship, we conducted $\varphi$ scans around the SL $(101)_{\mathrm{pc}}$ and $\mathrm{Si}(202)$ peaks, as shown in Figure $3 \mathrm{~d}$. For SLs grown on $\mathrm{CNO} / \mathrm{STO}$ and $\mathrm{rGO} / \mathrm{STO}$ templates, no clear peaks show up for the SLs, indicating inplane textured films. This is consistent with the in-plane random distribution of $\mathrm{CNO}$ and $\mathrm{rGO}$ nanosheets on silicon. In contrast, the SLs grown on M-STO, YCLS, and P-STO show four $(101)_{\mathrm{pc}}$ peaks located $45^{\circ}$ apart from the corresponding $\mathrm{Si}(202)$ peaks. Hence, the epitaxial relationship can be derived as: SL $[001]_{\mathrm{pc}} / / \mathrm{Si}[001]$ and SL $[100]_{\mathrm{pc}} / / \mathrm{Si}$ $[110]$. For the YCLS buffer, the cube-on-cube epitaxy with YSZ $[001] / / \mathrm{Si}[001]$ and YSZ $[110] / / \mathrm{Si}[110]$ is established after the scavenge reaction. ${ }^{31}$ Such epitaxial relationship is retained for $\mathrm{CeO}_{2}$ grown on YSZ (Figure S6 in the Supporting Information). However, a clear $45^{\circ}$ rotation of the lattice can be determined from RHEED (Figure $\mathrm{S} 1$ in the Supporting Information) when $\mathrm{LaNiO}_{3}$ is grown on $\mathrm{CeO}_{2}$. It is important to note that the thin $\mathrm{LaNiO}_{3}$ layer is inserted to ensure (001) growth for the overlying STO layer, ${ }^{39}$ since the direct growth of STO on $\mathrm{CeO}_{2}$ yields a preferential (110) growth. ${ }^{40}$ This can benefit from the negligible lattice mismatch between $\mathrm{LaNiO}_{3}$ $\left(a_{\mathrm{pc}}=3.84 \AA\right)$ and $\mathrm{CeO}_{2}(a / \sqrt{ } 2=3.83 \AA)$.

The strain states of LMO/STO SLs are evaluated based on the XRD $\theta-2 \theta$ scans (Figure 3a,b) and reciprocal space mappings (RSM) (Figure 4). In Figure 3a,b, the dotted lines mark the $(002)_{\mathrm{pc}}$ peak position of LMO/STO SL grown on an STO single crystal, where only epitaxial strain due to lattice mismatch is involved. As indicated from the RSM shown in Figure $4 \mathrm{a}$, the reference SL is fully strained to the STO single crystal. The in-plane and out-of-plane lattice constants can be
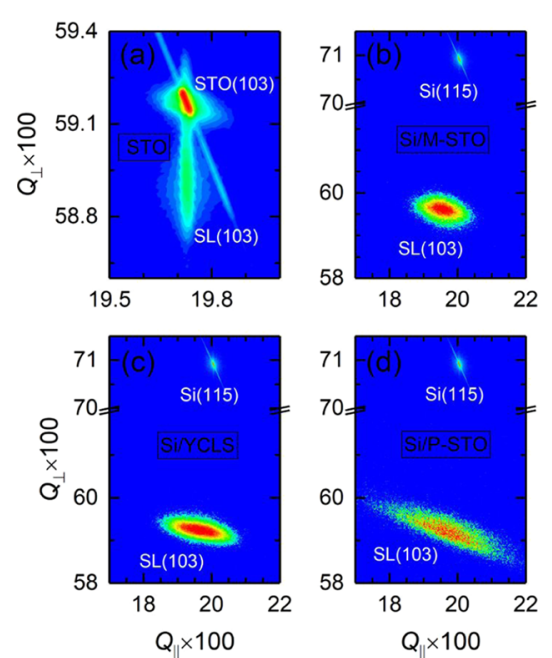

Figure 4. RSMs around $\mathrm{SL}(103)_{\mathrm{pc}}$ reflection taken from LMO/STO SLs grown on STO (a), Si/M-STO (b), Si/YCLS (c), and Si/P-STO (d). 
derived as $a=3.905 \AA$ and $c=3.922 \AA$, pointing to an in-plane compressive strain. For other SLs grown on $\mathrm{Si}$, thermal strain needs to be taken into account, which arises because of the large difference in thermal expansion coefficients $(\alpha)$ between oxides and silicon. ${ }^{41}$ Here, STO and LMO show $\alpha=8.7 \times$ $10^{-6} /{ }^{\circ} \mathrm{C}$ and $\alpha=11.2 \times 10^{-6} /{ }^{\circ} \mathrm{C}$, respectively, 3-4 times larger than $\alpha=2.6 \times 10^{-6} /{ }^{\circ} \mathrm{C}$ of $\mathrm{Si}^{4} .4,42$ As the SLs cool down from growth temperature $\left(700{ }^{\circ} \mathrm{C}\right)$, the less contracted $\mathrm{Si}$ substrate imparts a tensile thermal strain to oxide films by virtue of the clamping effect. For the $\mathrm{SL}$ on $\mathrm{Si} / \mathrm{CNO} / \mathrm{STO}$, the $(002)_{\mathrm{pc}}$ peak slightly shifts to higher angles and $c$ is calculated to be $3.912 \AA$. By assuming that the LMO/STO SL is strained to the $40 \mathrm{~nm}$ STO, this means an additional in-plane tensile strain plays a role as compared to the SL on STO. Since CNO has a smaller lattice constant $(3.86 \AA)$ than STO $(3.905 \AA)$ and LMO $(\sim 3.915 \AA$, extracted from a LMO single film grown on STO substrate using the same conditions as the SLs), ${ }^{24}$ such a tensile strain can only be linked to the thermal strain from the Si substrate. For the SL on Si/M-STO, the thermal strain effect is much more pronounced, yielding $a=3.942 \AA$ and $c=3.874 \AA$ (Figure $4 \mathrm{~b}$ ). Therefore, the functional layer LMO suffers a large tensile strain of $0.69 \%$ on $\mathrm{Si} / \mathrm{M}-\mathrm{STO}$, as compared to the small compressive strain of $0.26 \%$ when grown on the STO single crystal. For the SL on Si/YCLS, the thermal strain is partially compensated by the compressive epitaxial strain from $\mathrm{CeO}_{2} / \mathrm{LaNiO}_{3}$ layers, leading to $a=3.922$ $\AA$ and $c=3.901 \AA$ (Figure $4 c$ ). The SLs on $\mathrm{Si} / \mathrm{P}$-STO and $\mathrm{Si} /$ $\mathrm{rGO} / \mathrm{STO}$ show similar $c$ of 3.908 and $3.905 \AA$, respectively. The (103) reflection of SL grown on Si/P-STO (Figure 4d) shows a much more diffuse feature as compared to the SLs on $\mathrm{Si} / \mathrm{M}-\mathrm{STO}$ and $\mathrm{Si} / \mathrm{YCLS}$, testifying to a more severe in-plane mosaic spread. ${ }^{43}$ The main in-plane $a$ is determined to be $3.914 \AA$, slightly larger than the one on STO, indicating a weak contribution from thermal strain. The different amounts of thermal strain induced in LMO/STO SLs grown on various templates can be understood as follow. First, the thermal strain is imparted through the clamping effect from $\mathrm{Si}$, which is affected by the bonding in between the template and Si. Unlike the chemical bonds formed between $\mathrm{SiO}_{2}$ and STO or YSZ, $\mathrm{CNO}$ and rGO nanosheets are bonded to the Si surface by the relatively weak van der Waals force. Hence, thermal strain is less pronounced for the SLs grown on $\mathrm{Si} / \mathrm{CNO} / \mathrm{STO}$ and $\mathrm{Si} /$ $\mathrm{rGO} / \mathrm{STO}$. The suppressed thermal strain effects for the $\mathrm{CNO} / \mathrm{STO}$ template play a crucial role in the high-quality epitaxy of the overgrowing LMO/STO SLs. Second, defects, such as dislocations and grain boundaries (Figure S5 in the Supporting Information) can also partially release the thermal strain. This can explain the large difference in strain states between the SLs grown on M-STO and P-STO templates, considering more defects formed in the P-STO buffer. Third, the residual strain is also affected by the different thermal history during the preparation of various templates. For instance, elevating the growth temperature gives rise to a larger thermal strain for the overgrowing oxide films. ${ }^{41}$

The magnetic properties of LMO/STO SLs grown on various substrates are compared in Figure 5a,b. To ensure a ferromagnetic ground state, the LMO layer thickness is set to 6 uc in SLs, the critical thickness for ferromagnetism demonstrated in LMO single films. ${ }^{27}$ All SLs show ferromagnetic insulating ground states (transport data not shown). As can be seen from the temperature-dependent magnetization curves in Figure 5a, the SLs grown on an STO single crystal, $\mathrm{Si} / \mathrm{CNO} / \mathrm{STO}, \mathrm{Si} / \mathrm{M}-\mathrm{STO}$, and $\mathrm{Si} / \mathrm{YCLS}$ show
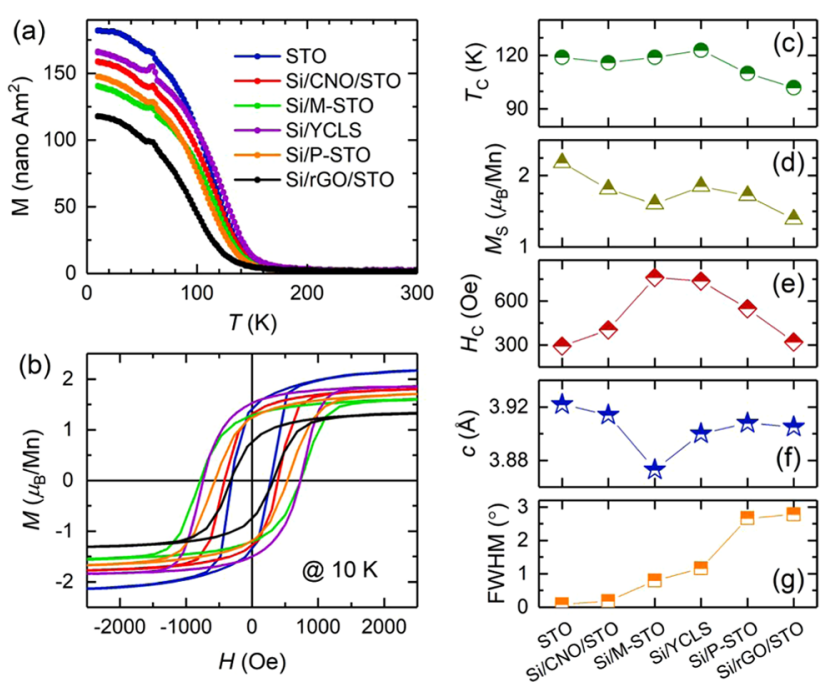

Figure 5. Temperature-dependent magnetization curves (a) and isothermal magnetic hysteresis loops at $10 \mathrm{~K}$ (b) measured from LMO/STO SLs. The temperature-dependent magnetization curves were measured by first cooling the samples down to $10 \mathrm{~K}$ in a field of $1 \mathrm{~T}$, then the data were recorded during warming up in a field of 0.1 $\mathrm{T}$. The magnetic field was applied along the $\mathrm{Si}[110]$ direction. (c-g) Summary of the magnetic and structural properties of LMO/STO SLs grown on various substrates.

virtually the same Curie temperature, $T_{\mathrm{C}} \sim 120 \pm 3 \mathrm{~K}$, which slightly decreases to 110 and $102 \mathrm{~K}$ for the SLs on $\mathrm{Si} / \mathrm{P}$-STO and $\mathrm{Si} / \mathrm{rGO} / \mathrm{STO}$, respectively. Figure $5 \mathrm{~b}$ shows the magnetic hysteresis loops measured at $10 \mathrm{~K}$. The reference SL grown on STO shows the largest saturation magnetization, $M_{\mathrm{S}} \sim 2.2 \mu_{\mathrm{B}} /$ Mn, comparable with a 20-uc-thick LMO single film grown under the same condition. This is different from a 6 uc LMO single film, which exhibits a drastically suppressed $M_{\mathrm{S}} \sim 1.6$ $\mu_{\mathrm{B}} / \mathrm{Mn}^{27}$ The preservation of ferromagnetism in SL architecture can be attributed to the removal of the notorious surface effect together with the modified $\mathrm{MnO}_{6}$ octahedral rotations close to LMO/STO interfaces. ${ }^{29,44}$ In comparison to the SL on STO, $M_{\mathrm{S}}$ is suppressed to some extent for SLs grown on silicon. Particularly, the SL on $\mathrm{Si} / \mathrm{rGO} / \mathrm{STO}$ shows a $M_{\mathrm{S}}$ of $\sim 1.3 \mu_{\mathrm{B}} / \mathrm{Mn}$, nearly $\sim 60 \%$ of the SL on STO. Here, the effects of in-plane magnetic anisotropy of LMO/STO SLs could be overestimated considering the comparable saturation fields between the textured and epitaxial SLs. Moreover, the SL on STO exhibits a slim loop with a coercive field, $H_{\mathrm{C}} \sim 290$ Oe, while broader loops with enhanced $H_{\mathrm{C}}$ have been observed for the SLs grown on silicon. The magnetic and structural properties of all SLs are further summarized in Figure $5 \mathrm{c}-\mathrm{g}$. Here $c$ and FWHM are taken as measures of the residual strain and crystallinity, respectively. In general, $T_{\mathrm{C}}$ and $M_{\mathrm{S}}$ show a trend of decline as FWHM increases, indicating that the degraded crystallinity weakens the magnetism. Surprisingly, the $\mathrm{SL}$ grown on $\mathrm{Si} / \mathrm{M}$-STO shows the largest $H_{\mathrm{C}} \sim 760$ Oe, while the most defective $\mathrm{SL}$ grown on $\mathrm{Si} / \mathrm{rGO} / \mathrm{STO}$ shows a small $H_{\mathrm{C}} \sim 320$ Oe. This means domain-wall pinning by defects is not the dominant factor determining $H_{\mathrm{C}}$ in these SLs. It is important to note that the $\mathrm{SL}$ on $\mathrm{Si} / \mathrm{M}$-STO receives the largest in-plane tensile strain, as inferred from the smallest $c$ in Figure 5f. The large tensile strain is able to induce antiferromagnetic phases in the ferromagnetic matrix of manganites via promoting in-plane orbital orders as well as enhancing Jahn-Teller distortions. ${ }^{45,46}$ Such antiferromagnetic 
clusters can act as pinning centers for the domain-wall motion and thus enhance $H_{\mathrm{C}}{ }^{47}$ Furthermore, this strain effect results in a smaller $M_{\mathrm{S}}$ for the $\mathrm{SL}$ on $\mathrm{Si} / \mathrm{M}$-STO than the one on $\mathrm{Si}$ / YCLS, although they have comparable crystalline quality. On the other hand, $T_{\mathrm{C}}$ seems to be hardly affected by the large tensile strain for the SL on Si/M-STO. This is probably linked to the unique magnetic cluster states proposed in LMO. ${ }^{28}$ Our findings are in line with the strain-dependent ferromagnetism of LMO single films, where $M_{\mathrm{S}}$ is largely suppressed for the more strained films grown on $\left(\mathrm{LaAlO}_{3}\right)_{0.3}\left(\mathrm{Sr}_{2} \mathrm{AlTaO}_{6}\right)_{0.7}$ and $\mathrm{LaAlO}_{3}$ as compared to the film grown on STO, but all three films show essentially the same onset temperature of magnetization. $^{48}$

\section{CONCLUSIONS}

To summarize, (001) single oriented LMO/STO SLs have been successfully integrated on silicon using various template techniques. The SLs grown on M-STO, YCLS, and P-STO templates show an epitaxial relationship with the underlying silicon substrate, while the SLs on CNO/STO and $\mathrm{rGO} / \mathrm{STO}$ are in-plane textured. Due to the small lattice mismatch between $\mathrm{CNO}$ and STO as well as less clamping from the Si substrate, the SL grown on $\mathrm{CNO} / \mathrm{STO}$ shows the highest crystallinity with a rocking curve FWHM of $\sim 0.18^{\circ}$. Welldefined SL peaks have been observed for the SLs grown on $\mathrm{CNO} / \mathrm{STO}, \mathrm{M}-\mathrm{STO}$, and YCLS templates, but are absent for the SLs on P-STO and $\mathrm{rGO} / \mathrm{STO}$ due to the degraded crystallinity. It is also found that the $\mathrm{SL}$ on $\mathrm{Si} / \mathrm{M}-\mathrm{STO}$ suffers the largest thermal strain from the $\mathrm{Si}$ substrate, giving rise to a strongly enhanced $H_{C}$ as compared to the reference SL on an STO single crystal. The multiple approaches of integrating oxide heterostructures on silicon presenting in this work may pave the way toward the practical applications of oxides in the future Si technology.

\section{ASSOCIATED CONTENT}

\section{SI Supporting Information}

The Supporting Information is available free of charge at https://pubs.acs.org/doi/10.1021/acsami.0c10579.

RHEED patterns for the growth of the YCLS multilayer on silicon (Figure S1) as well as the growth of STO on CNO (Figure S2) and rGO (Figure S3) nanosheets, RHEED oscillation of LMO/STO SLs grown on various substrates (Figure S4), cross-sectional scanning electron microscopy characterizations of the SLs on silicon (Figure S5), and XRD $\varphi$ scans of the YCLS multilayer (Figure S6) (PDF)

\section{AUTHOR INFORMATION}

\section{Corresponding Author}

Gertjan Koster - MESA+ Institute for Nanotechnology, University of Twente, 7500 AE Enschede, The Netherlands; ○ orcid.org/0000-0001-5478-7329; Email: g.koster@ utwente.nl

\section{Authors}

Binbin Chen - MESA+ Institute for Nanotechnology, University of Twente, 7500 AE Enschede, The Netherlands

Zoran Jovanovic - Advanced Materials Department, Jožef Stefan Institute, 1000 Ljubljana, Slovenia; Laboratory of Physics, Vinca Institute of Nuclear Sciences, University of Belgrade, 11000 Belgrade, Serbia
Stefan Abel - IBM Research-Zurich, 8803 Rüschlikon, Switzerland; Lumiphase AG, 8003 Zürich, Switzerland

Phu Tran Phong Le - MESA+ Institute for Nanotechnology, University of Twente, 7500 AE Enschede, The Netherlands; (1) orcid.org/0000-0003-1791-7184

Ufuk Halisdemir - MESA+ Institute for Nanotechnology, University of Twente, 7500 AE Enschede, The Netherlands

Mark Smithers - MESA+ Institute for Nanotechnology, University of Twente, 7500 AE Enschede, The Netherlands

Daniel Diaz-Fernandez - Advanced Materials Department, Jožef Stefan Institute, 1000 Ljubljana, Slovenia

Matjaž Spreitzer - Advanced Materials Department, Jožef Stefan Institute, 1000 Ljubljana, Slovenia

Jean Fompeyrine - IBM Research-Zurich, 8803 Rüschlikon, Switzerland; Lumiphase AG, 8003 Zürich, Switzerland

Guus Rijnders - MESA+ Institute for Nanotechnology, University of Twente, 7500 AE Enschede, The Netherlands

Complete contact information is available at:

https://pubs.acs.org/10.1021/acsami.0c10579

\section{Author Contributions}

B.C., P.T.P.L., and U.H. prepared the Si/YCLS and Si/CNO/ STO templates under the supervision of G.K. and G.R. Z.J. and D.D.-F. prepared the $\mathrm{Si} / \mathrm{rGO} / \mathrm{STO}$ and $\mathrm{Si} / \mathrm{P}-\mathrm{STO}$ templates under the supervision of M. Spreitzer S.A. prepared the Si/MSTO template under the supervision of J.F. B.C. performed the PLD growth, structural, and magnetic characterizations of LMO/STO SLs. M. Smithers conducted scanning electron microscopy measurements. B.C. and G.K. wrote the paper with inputs from all coauthors.

\section{Notes}

The authors declare no competing financial interest.

\section{ACKNOWLEDGMENTS}

This work is supported by the NWO's TOP-PUNT Grant (No. 718.016002), the international M-ERA.NET project SIOX (Project 4288), and the H2020 project ULPEC (Project 732642). M. Spreitzer acknowledges funding from the Slovenian Research Agency (Grants J2-9237 and P2-0091).

\section{REFERENCES}

(1) Ngai, J. H.; Walker, F. J.; Ahn, C. H. Correlated Oxide Physics and Electronics. Annu. Rev. Mater. Res. 2014, 44, 1-17.

(2) Schlom, D. G.; Chen, L. Q.; Pan, X.; Schmehl, A.; Zurbuchen, M. A. A Thin Film Approach to Engineering Functionality into Oxides. J. Am. Ceram. Soc. 2008, 91, 2429-2454.

(3) Hwang, H. Y.; Iwasa, Y.; Kawasaki, M.; Keimer, B.; Nagaosa, N.; Tokura, Y. Emergent Phenomena at Oxide Interfaces. Nat. Mater. 2012, 11, 103-113.

(4) Zubko, P.; Gariglio, S.; Gabay, M.; Ghosez, P.; Triscone, J.-M. Interface Physics in Complex Oxide Heterostructures. Annu. Rev. Condens. Matter Phys. 2011, 2, 141-165.

(5) Huang, Z.; Ariando; Wang, X. R.; Rusydi, A.; Chen, J.; Yang, H.; Venkatesan, T. Interface Engineering and Emergent Phenomena in Oxide Heterostructures. Adv. Mater. 2018, 30, No. 1802439.

(6) Xu, Z.; Hu, S.; Wu, R.; Wang, J. O.; Wu, T.; Chen, L. StrainEnhanced Charge Transfer and Magnetism at a Manganite/Nickelate Interface. ACS Appl. Mater. Interfaces 2018, 10, 30803-30810.

(7) Baek, S. H.; Eom, C. B. Epitaxial Integration of Perovskite-Based Multifunctional Oxides on Silicon. Acta Mater. 2013, 61, 2734-2750.

(8) Reiner, J. W.; Kolpak, A. M.; Segal, Y.; Garrity, K. F.; IsmailBeigi, S.; Ahn, C. H.; Walker, F. J. Crystalline Oxides on Silicon. Adv. Mater. 2010, 22, 2919-2938. 
(9) Schlom, D. G.; Guha, S.; Datta, S. Gate Oxides Beyond $\mathrm{SiO}_{2}$. MRS Bull. 2008, 33, 1017-1025.

(10) Park, B.-E.; Shouriki, S.; Tokumitsu, E.; Ishiwara, H. Fabrication of $\mathrm{PbZr}_{x} \mathrm{Ti}_{1-x} \mathrm{O}_{3}$ Films on Si Structures Using $\mathrm{Y}_{2} \mathrm{O}_{3}$ Buffer Layers. Jpn. J. Appl. Phys. 1998, 37, 5145-5149.

(11) Xiong, Y.; Wen, Q. Y.; Chen, Z.; Tian, W.; Wen, T. L.; Jing, Y. L.; Yang, Q. H.; Zhang, H. W. Tuning the Phase Transitions of $\mathrm{VO}_{2}$ thin Films on Silicon Substrates Using Ultrathin $\mathrm{Al}_{2} \mathrm{O}_{3}$ as Buffer Layers. J. Phys. D: Appl. Phys. 2014, 47, No. 455304.

(12) Basit, N. A.; Kim, H. K.; Blachere, J. Growth of Highly Oriented $\mathrm{Pb}(\mathrm{Zr}, \mathrm{Ti}) \mathrm{O}_{3}$ films on $\mathrm{MgO}$-Buffered Oxidized Si Substrates and Its Application to Ferroelectric Nonvolatile Memory Field-Effect Transistors. Appl. Phys. Lett. 1998, 73, 3941-3943.

(13) Fork, D. K.; Fenner, D. B.; Barton, R. W.; Phillips, J. M.; Connell, G. A. N.; Boyce, J. B.; Geballe, T. H. High Critical Currents in Strained Epitaxial $\mathrm{YBa}_{2} \mathrm{Cu}_{3} \mathrm{O}_{7-\delta}$ on Si. Appl. Phys. Lett. 1990, 57, $1161-1163$.

(14) McKee, R. A.; Walker, F. J.; Chisholm, M. F. Crystalline Oxides on Silicon: The First Five Monolayers. Phys. Rev. Lett. 1998, 81, No. 3014.

(15) Baek, S. H.; Park, J.; Kim, D. M.; Aksyuk, V. A.; Das, R. R.; Bu, S. D.; Felker, D. A.; Lettieri, J.; Vaithyanathan, V.; Bharadwaja, S. S. N.; Bassiri-Gharb, N.; Chen, Y. B.; Sun, H. P.; Folkman, C. M.; Jang, H. W.; Kreft, D. J.; Streiffer, S. K.; Ramesh, R.; Pan, X. Q.; TrolierMcKinstry, S.; Schlom, D. G.; Rzchowski, M. S.; Blick, R. H.; Eom, C. B. Giant Piezoelectricity on Si for Hyperactive MEMS. Science 2011, 334, 958-961.

(16) Bhaskar, U. K.; Banerjee, N.; Abdollahi, A.; Wang, Z.; Schlom, D. G.; Rijnders, G.; Catalan, G. A Flexoelectric Microelectromechanical System on Silicon. Nat. Nanotechnol. 2016, 11, 263-266.

(17) Park, J. W.; Bogorin, D. F.; Cen, C.; Felker, D. A.; Zhang, Y.; Nelson, C. T.; Bark, C. W.; Folkman, C. M.; Pan, X. Q.; Rzchowski, M. S.; Eom, C. B. Creation of a Two-Dimensional Electron Gas at an Oxide Interface on Silicon. Nat. Commun. 2010, 1, No. 94.

(18) Abel, S.; Stöferle, T.; Marchiori, C.; Rossel, C.; Rossell, M. D.; Erni, R.; Caimi, D.; Sousa, M.; Chelnokov, A.; Offrein, B. J.; Fompeyrine, J. A Strong Electro-Optically Active Lead-Free Ferroelectric Integrated on Silicon. Nat. Commun. 2013, 4, No. 1671.

(19) Abel, S.; Eltes, F.; Ortmann, J. E.; Messner, A.; Castera, P.; Wagner, T.; Urbonas, D.; Rosa, A.; Gutierrez, A. M.; Tulli, D.; Ma, P.; Baeuerle, B.; Josten, A.; Heni, W.; Caimi, D.; Czornomaz, L.; Demkov, A. A.; Leuthold, J.; Sanchis, P.; Fompeyrine, J. Large Pockels Effect in Micro- and Nanostructured Barium Titanate Integrated on Silicon. Nat. Mater. 2019, 18, 42-47.

(20) Nam, S. H.; Lee, W. J.; Kim, H. G. Oriented Growth of $\mathrm{SrTiO}_{3}$ Thin Films on Si Substrate by Radio Frequency Magnetron Sputtering. J. Phys. D: Appl. Phys. 1994, 27, 866-870.

(21) Klement, D.; Spreitzer, M.; Suvorov, D. Formation of a Strontium Buffer Layer on $\mathrm{Si}(001)$ by Pulsed-Laser Deposition through the $\mathrm{Sr} / \mathrm{Si}(001)(2 \times 3)$ Surface Reconstruction. Appl. Phys. Lett. 2015, 106, No. 071602.

(22) Spreitzer, M.; Klement, D.; Egoavil, R.; Verbeeck, J.; Kovač, J.; Založnik, A.; Koster, G.; Tendeloo, V. G.; Suvorov, D.; Rijnders, G. Growth Mechanism of Epitaxial $\mathrm{SrTiO}_{3}$ on a $(1 \times 2)+(2 \times 1)$ Reconstructed $\operatorname{Sr}(1 / 2 \mathrm{ML}) / \mathrm{Si}(001)$ Surface. J. Mater. Chem. C 2020, $8,518-527$.

(23) Diaz-Fernandez, D.; Spreitzer, M.; Parkelj, T.; Suvorov, D. Multi-Stage Pulsed Laser Deposition of High Quality Epitaxial UltraThin $\mathrm{SrTiO}_{3}$ on Si Substrates. Appl. Surf. Sci. 2018, 455, 227-235.

(24) Shibata, T.; Fukuda, K.; Ebina, Y.; Kogure, T.; Sasaki, T. OneNanometer-Thick Seed Layer of Unilamellar Nanosheets Promotes Oriented Growth of Oxide Crystal Films. Adv. Mater. 2008, 20, 231235.

(25) Nijland, M.; Thomas, S.; Smithers, M. A.; Banerjee, N.; Blank, D. H. A.; Rijnders, G.; Xia, J.; Koster, G.; Ten Elshof, J. E. Epitaxy on Demand. Adv. Funct. Mater. 2015, 25, 5140-5148.

(26) Lee, S. A.; Hwang, J. Y.; Km, E. S.; Kim, S. W.; Choi, W. S. Highly Oriented $\mathrm{SrTiO}_{3}$ Thin Film on Graphene Substrate. ACS Appl. Mater. Interfaces 2017, 9, 3246-3250.
(27) Wang, X. R.; Li, C. J.; Lü, W. M.; Paudel, T. R.; Leusink, D. P.; Hoek, M.; Poccia, N.; Vailionis, A.; Venkatesan, T.; Coey, J. M. D.; Tsymbal, E. Y.; Ariando; Hilgenkamp, H. Imaging and Control of Ferromagnetism in $\mathrm{LaMnO}_{3} / \mathrm{SrTiO}_{3}$ Heterostructures. Science 2015, 349, 716-719.

(28) Anahory, Y.; Embon, L.; Li, C. J.; Banerjee, S.; Meltzer, A.; Naren, H. R.; Yakovenko, A.; Cuppens, J.; Myasoedov, Y.; Rappaport, M. L.; Huber, M. E.; Michaeli, K.; Venkatesan, T.; Ariando; Zeldov, E. Emergent Nanoscale Superparamagnetism at Oxide Interfaces. Nat. Commun. 2016, 7, No. 12566.

(29) Zhai, X.; Cheng, L.; Liu, Y.; Schlepütz, C. M.; Dong, S.; Li, H.; Zhang, X.; Chu, S.; Zheng, L.; Zhang, J.; Zhao, A.; Hong, H.; Bhattacharya, A.; Eckstein, J. N.; Zeng, C. Correlating Interfacial Octahedral Rotations with Magnetism in $\left(\mathrm{LaMnO}_{3+\delta}\right)_{\mathrm{N}} /\left(\mathrm{SrTiO}_{3}\right)_{\mathrm{N}}$ Superlattices. Nat. Commun. 2014, 5, No. 4283.

(30) Marchiori, C.; Sousa, M.; Guiller, A.; Siegwart, H.; Locquet, J. P.; Fompeyrine, J.; Norga, G. J.; Seo, J. W. Thermal Stability of the $\mathrm{SrTiO}_{3} /(\mathrm{Ba}, \mathrm{Sr}) \mathrm{O}$ Stacks Epitaxially Grown on Si. Appl. Phys. Lett. 2006, 88, No. 072913.

(31) Lubig, A.; Buchal, C.; Guggi, D.; Jia, C. L.; Stritzker, B. Epitaxial Growth of Monoclinic and Cubic $\mathrm{ZrO}_{2}$ on $\mathrm{Si}(100)$ without Prior Removal of the Native $\mathrm{SiO}_{2}$. Thin Solid Films 1992, 217, 125-128.

(32) Nguyen, M. D.; Yuan, H.; Houwman, E. P.; Dekkers, M.; Koster, G.; Ten Elshof, J. E.; Rijnders, G. Highly Oriented Growth of Piezoelectric Thin Films on Silicon Using Two-Dimensional Nanosheets as Growth Template Layer. ACS Appl. Mater. Interfaces 2016, 8, 31120-31127.

(33) Jovanović, Z.; Spreitzer, M.; Kovač, J.; Klement, D.; Suvorov, D. Silicon Surface Deoxidation Using Strontium Oxide Deposited with the Pulsed Laser Deposition Technique. ACS Appl. Mater. Interfaces 2014, 6, 18205-18214.

(34) Koster, G.; Rijnders, G. In Situ Characterization of Thin Film Growth; Woodhead Publishing: Cambridge, UK, 2012.

(35) Du, Y.; Zhang, K. H. L.; Varga, T.; Chambers, S. A. Reflection High-Energy Electron Diffraction Beam-Induced Structural and Property Changes on $\mathrm{WO}_{3}$ Thin Films. Appl. Phys. Lett. 2014, 105, No. 051606.

(36) Chang, J.; Park, Y. S.; Lee, J. W.; Kim, S. K. Layer-by-Layer Growth and Growth-Mode Transition of $\mathrm{SrRuO}_{3}$ Thin Films on Atomically Flat Single-Terminated $\mathrm{SrTiO}_{3}$ (111) Surfaces. J. Cryst. Growth 2009, 311, 3771-3774.

(37) Scigaj, M.; Chao, C. H.; Gázquez, J.; Fina, I.; Moalla, R.; SaintGirons, G.; Chisholm, M. F.; Herranz, G.; Fontcuberta, J.; Bachelet, R.; Sánchez, F. High Ferroelectric Polarization in c-Oriented $\mathrm{BaTiO}_{3}$ Epitaxial Thin Films on $\mathrm{SrTiO}_{3} / \mathrm{Si}(001)$. Appl. Phys. Lett. 2016, 109, No. 122903.

(38) Lyu, J.; Fina, I.; Solanas, R.; Fontcuberta, J.; Sánchez, F. Tailoring Lattice Strain and Ferroelectric Polarization of Epitaxial $\mathrm{BaTiO}_{3}$ Thin Films on Si(001). Sci. Rep. 2018, 8, No. 495.

(39) Scigaj, M.; Dix, N.; Fina, I.; Bachelet, R.; Warot-Fonrose, B.; Fontcuberta, J.; Sánchez, F. Ultra-Flat $\mathrm{BaTiO}_{3}$ Epitaxial Films on $\mathrm{Si}(001)$ with Large Out-of-Plane Polarization. Appl. Phys. Lett. 2013, 102, No. 112905.

(40) Yamada, T.; Wakiya, N.; Shinozaki, K.; Mizutani, N. Epitaxial Growth of $\mathrm{SrTiO}_{3}$ Films on $\mathrm{CeO}_{2} /$ Yttria-Stabilized Zirconia/Si(001) with $\mathrm{TiO}_{2}$ Atomic Layer by Pulsed-Laser Deposition. Appl. Phys. Lett. 2003, 83, 4815.

(41) Zhang, L.; Yuan, Y.; Lapano, J.; Brahlek, M.; Lei, S.; Kabius, B.; Gopalan, V.; Engel-Herbert, R. Continuously Tuning Epitaxial Strains by Thermal Mismatch. ACS Nano 2018, 12, 1306-1312.

(42) Srilomsak, S.; Schilling, D. P.; Anderson, H. U. Thermal Expansion Studies on Cathode and Interconnect Oxides. Proc. Electrochem. Soc. 1989, 140, 129-140.

(43) Wang, Z.; Chen, Z.; Mei, A. B.; Bai, X.; Kourkoutis, L. F.; Muller, D. A.; Schlom, D. G. Growth of $\mathrm{LaAlO}_{3}$ on Silicon via an Ultrathin $\mathrm{SrTiO}_{3}$ Buffer Layer by Molecular-Beam Epitaxy. J. Vac. Sci. Technol., A 2017, 36, No. 021507.

(44) Wu, L.; Li, C.; Chen, M.; Zhang, Y.; Han, K.; Zeng, S.; Liu, X.; Ma, J.; Liu, C.; Chen, J.; Zhang, J.; Ariando; Venkatesan, T. V.; 
Pennycook, S. J.; Coey, J. M. D.; Shen, L.; Ma, J.; Wang, X. R.; Nan, C. W. Interface-Induced Enhancement of Ferromagnetism in Insulating $\mathrm{LaMnO}_{3}$ Ultrathin Films. ACS Appl. Mater. Interfaces 2017, 9, 44931-44937.

(45) Fang, Z.; Solovyev, I. V.; Terakura, K. Phase Diagram of Tetragonal Manganites. Phys. Rev. Lett. 2000, 84, No. 3169.

(46) Lee, J. H.; Delaney, K. T.; Bousquet, E.; Spaldin, N. A.; Rabe, K. M. Strong Coupling of Jahn-Teller Distortion to OxygenOctahedron Rotation and Functional Properties in Epitaxially Strained Orthorhombic $\mathrm{LaMnO}_{3}$. Phys. Rev. B 2013, 88, No. 174426.

(47) Sharma, M.; Gazquez, J.; Varela, M.; Schmitt, J.; Leighton, C. Coercivity Enhancement Driven by Interfacial Magnetic Phase Separation in $\mathrm{SrTiO}_{3}(001) / \mathrm{Nd}_{0.5} \mathrm{Sr}_{0.5} \mathrm{CoO}_{3}$. Phys. Rev. B 2011, 84, No. 024417

(48) Niu, W.; Liu, W.; Gu, M.; Chen, Y.; Zhang, X.; Zhang, M.; Chen, Y.; Wang, J.; Du, J.; Song, F.; Pan, X.; Pryds, N.; Wang, X.; Wang, P.; Xu, Y.; Chen, Y.; Zhang, R. Direct Demonstration of the Emergent Magnetism Resulting from the Multivalence $\mathrm{Mn}$ in a $\mathrm{LaMnO}_{3}$ Epitaxial Thin Film System. Adv. Electron. Mater. 2018, 4, No. 1800055. 